\title{
The Bonn nuclear quark model revisited
}

\author{
Constança Providência, João da Providência, Flávio Cordeiro \\ Departamento de Física, Universidade de Coimbra, \\ P-3004-516 Coimbra, Portugal \\ Masatoshi Yamamura \\ Department of Pure and Applied Physics, Faculty of Engineering Science, \\ Kansai University, Suita 564-8680, Japan \\ Yasuhiko Tsue, Seiya Nishiyama \\ Physics Division, Faculty of Science, Kochi University,
} Kochi 780-8520, Japan

August 10, 2018

\begin{abstract}
We present the exact solutions to the equations of the lowest energy states of the colored and color-symmetric sectors of the Bonn quark model, which is $S U(3)$ symmetric and is defined in terms of an effective pairing force with $s u(4)$ algebraic structure. We show that the groundstate of the model is not color symmetrical except for a narrow interval in the range of possible quark numbers. We also study the performance of the Glauber coherent state, as well as of superconducting states of the BCS type, with respect to the description, not only of the absolute (colored) groundstate, but also of the minimum energy state of the color-symmetrical sector, finding that it is remarkably good. We use the model to discuss, in a schematic context, some controversial aspects of the conventional treatment of color superconductivity.
\end{abstract}

\section{Introduction}

The so-called Bonn model, which is here reinvestigated, has been proposed by Petry et al. [1] some years ago for the description of the nucleus as a system of interacting quarks, which, according to the current belief, is based on the QCD field theory. The model is inspired by the famous seniority model of nuclear physics that explains the superconducting features of nuclear structure, namely, a characteristic gap in some spectra associated with a pairing force (cf. [2], p. 121). An important ingredient in the Bonn model is an attractive pairing force, acting between quarks of different colors, that suppresses physically undesirable degeneracies of the quark system. Although the model is too schematic to be realistic, it qualitatively accounts for some features of nuclear physics. This model was originally aimed at explaining the formation of color neutral triplets, that is, clustering of quarks into nucleons. One of the reasons why it is not realistic is that it contains a colored sector, to 
which the groundstate belongs. Indeed, the existence of the colored sector is a physically undesirable artifact of the model, since, according to QCD, there are no colored states. Nevertheless, the model brings color symmetrical states rather close to the groundstate. This is quite remarkable since the involved interaction is a twobody force, which is naturally associated with two-body correlations, but not with three-body-correlations.

The model is particularly interesting because it is exactly soluble, so that it is appropriate for testing certain classes of approximation techniques. In this note we explore this aspect, by studying the performance of the Glauber coherent state (GCS) and of color superconducting states of the BCS type. It is found, quite remarkably, that the GCS and the BCS states provide good descriptions, not only of the absolute (colored) groundstate, but also of the minimum energy state of the color-symmetrical sector. We emphasize, however, that in order to properly describe the physically important color-symmetrical sector, a modified BCS approach, based on the generalized Bogoliubov transformation, which treats the three colors on the same footing [4], is needed.

A BCS color superconducting state $\left|\Phi_{B C S}\right\rangle$ is characterized by an order parameter $\widetilde{\Delta}$ which is a triplet belonging to the $\overline{3}$ representation of $S U(3)$, i.e. $\widetilde{\Delta}=$ $\left(\Delta_{1}, \Delta_{2}, \Delta_{3}\right)$. The starting point for the color neutral version of the BCS approach, considered in Ref. [4], is the assumption $\Delta_{1}=\Delta_{2}=\Delta_{3}=\Delta$, but, by a convenient color rotation, this may be turned into $\Delta_{1}=\sqrt{3} \Delta, \Delta_{2}=\Delta_{3}=0$, which is the starting assumption for the standard approach considered in Refs. [5, 6, 7]. Clearly, the groundstate energy remains unchanged. These two assumptions are equivalent if one imposes the restrictive condition

$$
\left\langle\Phi_{B C S}\left|S_{\Lambda_{k}}\right| \Phi_{B C S}\right\rangle=0, \quad k=1, \cdots, 8,
$$

where $S_{\Lambda_{k}}$ are the $S U(3)$ generators associated with the Gell Mann matrices $\Lambda_{k}$. On the other hand, for the standard BCS approach, either these relations are ignored altogether or, at most, the condition

$$
\left\langle\Phi_{B C S}\left|S_{\Lambda_{3}}\right| \Phi_{B C S}\right\rangle=\left\langle\Phi_{B C S}\left|S_{\Lambda_{8}}\right| \Phi_{B C S}\right\rangle=0
$$

is imposed through color dependent chemical potentials [7]. Since the condition (2) is not stable under color rotations, this is not enough, if our goal is the description of the color-symmetric states. In effective models of the NJL type, an un-physical colored sector coexists with the physically relevant color-symmetric sector, whose states are color singlets. As shown in Ref. [4, the colored sectors of the Bonn model, or of the NJL model, are described when the restrictive conditions (11) are altogether disregarded. On the other hand, the novel approach presented in Ref. [4] allows for the description of the physically relevant colorless sector.

It is well known that the dynamics of many-fermion systems may be described in terms of bosons. In the collective model of Bohr and Mottelson (cf. [2], p. 9), bosons were introduced through the quantization of the oscillations of a liquid drop to describe excitations of nuclei. In the theory of plasma oscillations, excited states of the electron gas are described by the so called random phase approximation which is nothing else than particle-hole pairs being approximated by bosons. The physical interpretation of the Schwinger bosons in the case of the Bonn model is an interesting problem which remains open.

We will present the exact solutions to the equations of the lowest energy states of the colored and color-symmetrical sectors of the of the Bonn quark model, and 
will show that the groundstate of the model is not color-symmetrical except for a narrow interval in the range of possible quark numbers. We will next study the performance of the Glauber coherent state with respect to the description, not only of the absolute (colored) groundstate, but also of the minimum energy state of the color-symmetrical sector. The color-symmetric sector of the Bonn model, characterized by its color singlet-ness, will receive a special attention. In particular, we will present its description, in terms of coherent states, such as color neutral Glauber coherent states, or superconducting states of the generalized BCS type introduced in [4].

\section{The model}

We wish to investigate the schematic nuclear model which has been proposed by H.R. Petry et al. [1, and further discussed by Pittel et al. [3], among others. Its Hamiltonian reads

$$
H=G \sum_{j=1}^{3} S^{i} S_{i}
$$

where

$$
S^{1}=S_{1}^{\dagger}=\sum_{m} c_{2 m}^{\dagger} c_{3 \tilde{m}}^{\dagger}, \quad S^{2}=S_{2}^{\dagger}=\sum_{m} c_{3 m}^{*} c_{1 \tilde{m}}^{\dagger}, \quad S^{3}=S_{3}^{\dagger}=\sum_{m} c_{1 m}^{\dagger} c_{2 \tilde{m}}^{\dagger} .
$$

Here, $c_{i m}^{\dagger}$ are fermion creation operators, the indexes $i, m$ denoting the color and the remaining single particle quantum numbers. The quantum number $\tilde{m}$ is obtained from $m$ by time reversal, so that $\tilde{\tilde{m}}=m$, or, more precisely, in a standard notation,

$$
c_{i \tilde{m}}^{\dagger}=(-1)^{j_{s}-m} c_{i,-m}^{\dagger} .
$$

Clearly, the operators $S^{i}, S_{i}$ generate an $s u(4)$ algebra. Indeed we have

$$
\left[S^{i}, S^{j}\right]=0, \quad\left[S^{i}, S_{j}\right]=S_{i}^{j}=-\sum_{m} c_{j m}^{\dagger} c_{i m}+\delta_{i j}\left(\sum_{k m} c_{k m}^{\dagger} c_{k m}-2 \Omega\right),
$$

where $2 \Omega$ denotes the level degeneracy, for fixed color, that is, the total number of single particle states beyond color. Moreover, the following relations, which close the $s u(4)$ algebra, are satisfied.

$$
\left[S_{i}^{j}, S^{k}\right]=\delta_{i j} S^{k}+\delta_{j k} S^{i}, \quad\left[S_{i}^{j}, S_{l}^{k}\right]=\delta_{j l} S_{i}^{k}-\delta_{i k} S_{l}^{j} .
$$

\section{Schwinger realization}

We use the Schwinger realization of $s u(4)$ in terms of 8 bosons, $a_{1}, a_{2}, a_{3}, a, b_{1}, b_{2}, b_{3}, b$, which has been proposed by Yamamura et al. [8], and reads

$$
\begin{aligned}
& S^{i}=a_{i}^{\dagger} b+a^{\dagger} b_{i}, \quad S_{i}=b^{\dagger} a_{i}+b_{i}^{\dagger} a, \\
& S_{j}^{i}=\left[S^{j}, S_{i}\right]=a_{j}^{\dagger} a_{i}-b_{i}^{\dagger} b_{j}+\delta_{i j}\left(a^{\dagger} a-b^{\dagger} b\right) .
\end{aligned}
$$

It may be easily checked that this realization preserves the algebra of the operators $S^{j}, S_{i}, S_{i}^{j}$, that is, the commutation relations in (5), (6) are satisfied. 
The transformed Hamiltonian reads

$$
\begin{aligned}
H= & G \sum_{i=1}^{3}\left(a_{i}^{\dagger} b^{\dagger} a_{i} b+a^{\dagger} b_{i}^{\dagger} a b_{i}+a_{i}^{\dagger} b_{i}^{\dagger} a b+a^{\dagger} b^{\dagger} a_{i} b_{i}\right) \\
& +G \sum_{i=1}^{3}\left(a_{i}^{\dagger} a_{i}+a^{\dagger} a\right) .
\end{aligned}
$$

This Hamiltonian admits the following constants of motion

$$
\begin{aligned}
& L_{i}=b_{i}^{\dagger} b_{i}-a_{i}^{\dagger} a_{i}, \quad i=1,2,3, \quad L=b^{\dagger} b-a^{\dagger} a, \\
& Q_{a}=\left(\sum_{i=1}^{3} a_{i}^{\dagger} a_{i}\right)+b^{\dagger} b, \quad Q_{b}=\left(\sum_{i=1}^{3} b_{i}^{\dagger} b_{i}\right)+a^{\dagger} a .
\end{aligned}
$$

The constant of motion $K=-\sum_{i=1}^{3}\left(L_{i}+L\right)$ is related to the number $N$ of quarks. From (5), we have

$$
K=2 N-6 \Omega \text {. }
$$

Moreover, $Q_{a}+Q_{b}$ commutes with all the generators of the algebra and so its eigenvalues characterize the physically relevant irreducible representations, being then

$$
S=Q_{a}+Q_{b}=2 \Omega
$$

We discuss now the construction of the lowest weight state. We will not consider states containing broken pairs. This means that our discussion is restricted to states $|\Phi\rangle$ such that the numbers of occupied single particle levels of the types $|i, m\rangle$ and $|j, \tilde{m}\rangle$ are the same, for any $i, j$ not necessarily different. Of course it is important to discuss also the case of broken pairs. This point will be considered elsewhere. The minimum weight state of the symmetric representation reads

$$
|\Omega\rangle=b^{\dagger 2 \Omega}|0\rangle,
$$

and satisfies

$$
S_{i}|\Omega\rangle=S_{i}^{j}|\Omega\rangle=0, \quad S_{i}^{i}|\Omega\rangle=-2 \Omega|\Omega\rangle, \quad\left(S^{i}\right)^{2 \Omega}|\Omega\rangle=(2 \Omega) ! a_{i}^{\dagger 2 \Omega}|0\rangle .
$$

The state $|\Omega\rangle$ is the boson image of the Fermion vacuum. In order to discuss asymmetric representations, we also consider the state:

$$
\left|\Omega, \Omega_{3}^{\prime}\right\rangle=b^{\dagger 2\left(\Omega-\Omega_{3}^{\prime}\right)} b_{3}^{\dagger}{ }^{2 \Omega_{3}^{\prime}}|0\rangle
$$

It satisfies:

$$
\begin{aligned}
& S_{i}\left|\Omega, \Omega_{3}^{\prime}\right\rangle=S_{1}^{2}\left|\Omega, \Omega_{3}^{\prime}\right\rangle=S_{2}^{1}\left|\Omega, \Omega_{3}^{\prime}\right\rangle=S_{1}^{3}\left|\Omega, \Omega_{3}^{\prime}\right\rangle=S_{2}^{3}\left|\Omega, \Omega_{3}^{\prime}\right\rangle=0, i=1,2,3, \\
& S_{1}^{1}\left|\Omega, \Omega_{3}^{\prime}\right\rangle=S_{2}^{2}\left|\Omega, \Omega_{3}^{\prime}\right\rangle=-2\left(\Omega-\Omega_{3}^{\prime}\right)\left|\Omega, \Omega_{3}^{\prime}\right\rangle, \quad S_{3}^{3}\left|\Omega, \Omega_{3}^{\prime}\right\rangle=-2 \Omega\left|\Omega, \Omega_{3}^{\prime}\right\rangle, \\
& \left(S^{i}\right)^{2\left(\Omega-\Omega^{\prime}\right)}\left|\Omega, \Omega_{3}^{\prime}\right\rangle=\left(2 \Omega-2 \Omega^{\prime}\right) ! a_{i}^{\dagger}{ }^{2\left(\Omega-\Omega_{3}^{\prime}\right)} b_{3}^{\dagger}{ }^{2 \Omega_{3}^{\prime}}|0\rangle, i=1,2, \\
& \left(S^{3}\right)^{2 \Omega}\left|\Omega, \Omega_{3}^{\prime}\right\rangle=(2 \Omega) ! a_{3}^{\dagger 2\left(\Omega-\Omega_{3}^{\prime}\right)} a^{\dagger \Omega_{3}^{\prime}}|0\rangle .
\end{aligned}
$$

The state $\left|\Omega, \Omega_{3}^{\prime}\right\rangle$ is the boson image of the fermionic state $\prod_{m=1}^{\Omega_{3}^{\prime}} c_{3 m}^{\dagger} c_{3 \tilde{m}}^{\dagger}\left|0_{F}\right\rangle$. The generators $S_{i}^{j}$ commute with $H$. They generate an $s u(3)$ sub-algebra of $s u(4)$. Thus, 
$H$ has $s u(3)$ symmetry. It may be easily shown that the states $\left(S^{i}\right)^{p}|\Omega\rangle$ (containing $2 p$ quarks) and $\left(S^{3}\right)^{p}\left|\Omega, \Omega_{3}^{\prime}\right\rangle_{\Omega_{3}^{\prime}=\Omega}$ (containing $2 p+2 \Omega$ quarks) are eigenstates of $H$,

$$
\begin{aligned}
H\left(S^{i}\right)^{p}|\Omega\rangle & =G p(2 \Omega+1-p)\left(S^{i}\right)^{p}|\Omega\rangle, \\
H\left(S^{3}\right)^{p}\left|\Omega, \Omega_{3}^{\prime}\right\rangle_{\Omega_{3}^{\prime}=\Omega} & =G p(2 \Omega+3-p)\left(S^{3}\right)^{p}\left|\Omega, \Omega_{3}^{\prime}\right\rangle_{\Omega_{3}^{\prime}=\Omega} .
\end{aligned}
$$

For $0<\Omega_{3}^{\prime}<\Omega$ we find

$$
\frac{\left\langle p, \Omega, \Omega_{3}^{\prime}|H| p, \Omega, \Omega_{3}^{\prime}\right\rangle}{\left\langle p, \Omega, \Omega_{3}^{\prime} \mid p, \Omega, \Omega_{3}^{\prime}\right\rangle}=G p\left(2 \Omega+1+2 \frac{\Omega_{3}^{\prime}}{\Omega}-p\right),
$$

where $\left|p, \Omega, \Omega_{3}^{\prime}\right\rangle=\left(S^{3}\right)^{p}\left|\Omega, \Omega_{3}^{\prime}\right\rangle$. This state contains $N=2\left(p+\Omega_{3}^{\prime}\right)$ quarks. For fixed $\Omega_{3}^{\prime}$, this expectation value, as a function of $N$, is a parabola. The envelope of these parabolas is the parabolic arc $G \frac{\left(N+\Omega+2 \Omega^{2}\right)^{2}}{4 \Omega(2+\Omega)}$. Therefore, for $2 \Omega \leq N \leq 4 \Omega$, the groundstate energy is, according to the Born approximation, $G \frac{\left(N+\Omega+2 \Omega^{2}\right)^{2}}{4 \Omega(2+\Omega)} \approx$ $G\left(\Omega^{2}-\Omega+N\right)$. For $N \leq 2 \Omega$, the groundstate energy is $G N(4 \Omega+2-N) / 4$. For $4 \Omega \leq N \leq 6 \Omega$, the groundstate energy is $G(N-2 \Omega)(6 \Omega+6-N) / 4$. The Bonn model was originally devised as a model for the formation of triples (clustering of quarks into nucleons). However, the tendency for the formation of triplets is not dominant. If it were, the groundstate would have $S U(3)$ symmetry, which is not the case. The investigation of the groundstate energy beyond the Born approximation will be carried out in Sections 5 and 6 .

\section{Glauber coherent state}

It is believed that color superconductivity plays an important role in high density matter, such as may be found in the core of neutron stars [5, 6, 7]. When the BCS theory is applied to the superconducting phase of quark matter, it is found that the BCS state violates color charge neutrality [7]. This undesirable feature is attributed to the BCS theory itself. However, in the model (3) which is used here, or in models of the NJL type [7], color symmetry breaking states, which are unphysical, coexist with the physically relevant states, which are color symmetric. In [4], it was shown that superconducting BCS states describe well the lowest energy colored and color-symmetric states of the Bonn model. There, it was also shown that the description of the physically relevant color-symmetric sector requires a modified version of the conventional BCS approach based on the generalized Bogoliubov transformation. Glauber coherent states have some analogies with BCS states. So, it may be interesting to investigate their performance. The GCS may be written

$$
|\Phi\rangle=\left|\alpha_{3}, \alpha, \beta_{3}, \beta\right\rangle=\exp \left(\alpha_{3} a_{3}^{\dagger}+\alpha a^{\dagger}+\beta_{3} b_{3}^{\dagger}+\beta b^{\dagger}\right)|0\rangle .
$$

The Hamiltoninan expectation value reads

$$
\begin{aligned}
\mathcal{E}=\frac{\langle\Phi|H| \Phi\rangle}{\langle\Phi \mid \Phi\rangle} & =G\left(\alpha_{3}^{*} \beta^{*} \alpha_{3} \beta+\alpha^{*} \beta_{3}^{*} \alpha \beta_{3}+\alpha_{3}^{*} \beta_{3}^{*} \alpha \beta+\alpha^{*} \beta^{*} \alpha_{3} \beta_{3}\right) \\
& +G\left(\alpha_{3}^{*} \alpha_{3}+3 \alpha^{*} \alpha\right) .
\end{aligned}
$$

The expectation values of the constants of motion read

$$
\begin{aligned}
& \mathcal{Q}_{a}=\left\langle\Phi\left|Q_{a}\right| \Phi\right\rangle=\alpha_{3}^{*} \alpha_{3}+\beta^{*} \beta=2\left(\Omega-\Omega^{\prime}\right), \\
& \mathcal{Q}_{b}=\left\langle\Phi\left|Q_{b}\right| \Phi\right\rangle=\beta_{3}^{*} \beta_{3}+\alpha^{*} \alpha=2 \Omega^{\prime} \\
& \mathcal{K}=\langle\Phi|K| \Phi\rangle=\alpha_{3}^{*} \alpha_{3}-\beta_{3}^{*} \beta_{3}+3\left(\alpha^{*} \alpha-\beta^{*} \beta\right) .
\end{aligned}
$$




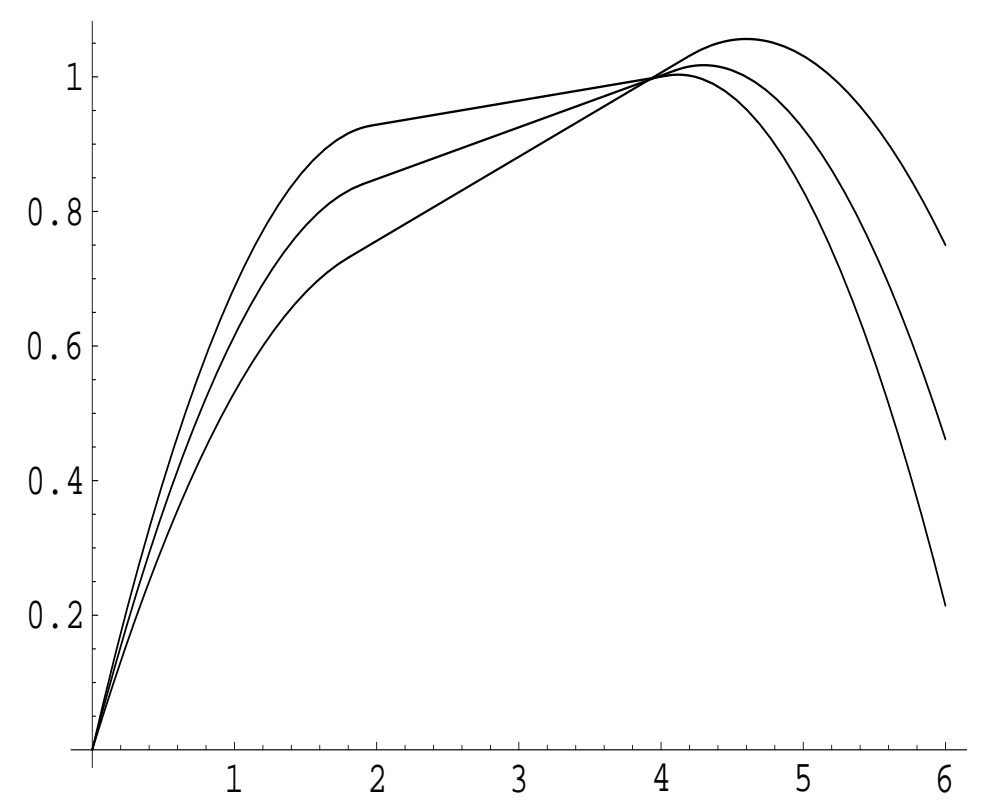

Figure 1: Groundstate energy, divided by $G \Omega(\Omega+3)$, vs. $N / \Omega$, according to the Glauber coherent state, for $\Omega=5$ (lower curve for $N / \Omega \leq 4$ ), $\Omega=10$ (middle curve for $N / \Omega \leq 4$ ), and $\Omega=20$ (upper curve for $N / \Omega \leq 4$ ). It almost coincides with the "exact" result, which will be presented later.

We may chose $\arg \left(\alpha_{3}\right)+\arg \left(\beta_{3}\right)-\arg (\alpha)-\arg (\beta)=0$. Then, we are led to minimize

$$
\mathcal{E}=G\left(\left|\alpha_{3}\right|^{2}|\beta|^{2}+|\alpha|^{2}\left|\beta_{3}\right|^{2}+2\left|\alpha_{3}\right||\beta|\left|\beta_{3}\right||\alpha|+\left|\alpha_{3}\right|^{2}+3|\alpha|^{2}\right),
$$

with respect to $\left|\alpha_{3}\right|,\left|\beta_{3}\right|,|\alpha|,|\beta|$, under the constraints

$$
\begin{gathered}
2 \Omega=\left|\alpha_{3}\right|^{2}+\left|\beta_{3}\right|^{2}+|\alpha|^{2}+|\beta|^{2} \\
2 N-6 \Omega=\left|\alpha_{3}\right|^{2}-\left|\beta_{3}\right|^{2}+3\left(|\alpha|^{2}-|\beta|^{2}\right) .
\end{gathered}
$$

\subsection{Computational details}

If $N \leq 2 \Omega-1$, the minimum energy occurs for $\left|\beta_{3}\right|=|\alpha|=0$. Then

$$
\mathcal{E}_{0}=G\left(\left|\alpha_{3}\right|^{2}|\beta|^{2}+\left|\alpha_{3}\right|^{2}\right)
$$

with $2 \Omega=\left|\alpha_{3}\right|^{2}+|\beta|^{2}, 2 N-6 \Omega=\left|\alpha_{3}\right|^{2}-3|\beta|^{2}$, so that $\left|\alpha_{3}\right|^{2}=N / 2,|\beta|^{2}=2 \Omega-N / 2$, and

$$
\mathcal{E}_{0}=\frac{G}{4} N(4 \Omega-N+2) \text {. }
$$

If $4 \Omega+1 \leq N \leq 6 \Omega$, the minimum energy occurs for $|\beta|=\left|\alpha_{3}\right|=0$. Then

$$
\mathcal{E}_{0}=G\left(|\alpha|^{2}\left|\beta_{3}\right|^{2}+3|\alpha|^{2}\right)
$$

with $2 \Omega=|\alpha|^{2}+\left|\beta_{3}\right|^{2}, 2 N-6 \Omega=3|\alpha|^{2}-\left|\beta_{3}\right|^{2}$, so that $|\alpha|^{2}=N / 2-\Omega,\left|\beta_{3}\right|^{2}=$ $3 \Omega-N / 2$, and

$$
\mathcal{E}_{0}=\frac{G}{4}(N-2 \Omega)(6 \Omega+6-N) .
$$


If $2 \Omega-1 \leq N \leq 4 \Omega+1$, the minimum energy occurs for $|\beta| \approx\left|\alpha_{3}\right| \neq 0,\left|\beta_{3}\right| \approx|\alpha| \neq 0$. This approximate result becomes exact in the limit of large $\Omega$. Assuming $|\beta|=\left|\alpha_{3}\right|$, $\left|\beta_{3}\right|=|\alpha|$ we may write

$$
\mathcal{E}_{0}=G\left(\left(|\alpha|^{2}+|\beta|^{2}\right)^{2}+|\beta|^{2}+3|\alpha|^{2}\right),
$$

with $2 \Omega=2|\alpha|^{2}+2|\beta|^{2}, 2 N-6 \Omega=2\left(|\alpha|^{2}-|\beta|^{2}\right)$, so that $|\alpha|^{2}=N / 2-\Omega$, $|\beta|^{2}=2 \Omega-N / 2$, and $\mathcal{E}_{0} \approx G\left(\Omega^{2}-\Omega+N\right)$. If first order corrections are consistently taken into account we find

$$
\mathcal{E}_{0}=G\left(\Omega^{2}-\Omega+N+\frac{1}{4}\right) .
$$

Indeed, it may be seen that for fixed values of $\Omega$ and $\Omega^{\prime}=\left|\beta_{3}\right|^{2}+|\alpha|^{2}$, such that $0<\Omega^{\prime}<\Omega$, the minimum energy $\mathcal{E}$ as a function of $N$, lies on a similar parabola to the one in eq. (A), but properly translated, so that it is tangent to the line described by eq. (C).

In short, the groundstate energy reads

$$
\begin{array}{ll}
\mathcal{E}_{0}=\frac{G}{4} N(4 \Omega-N+2), \quad \text { if } & 0 \leq N \leq 2 \Omega-1 ; \\
\mathcal{E}_{0}=G\left(\Omega^{2}-\Omega+N+\frac{1}{4}\right), & \text { if } \quad 2 \Omega-1 \leq N \leq 4 \Omega+1 ; \\
\mathcal{E}_{0}=\frac{G}{4}(N-2 \Omega)(6 \Omega-N+6), & \text { if } \quad 4 \Omega+1 \leq N,
\end{array}
$$

which is very close to the exact result. This is quite remarkable. Rather than being regarded as a model for the formation of triples which are invariant under $S U(3)$, the Bonn model may be viewed as a model for color superconductivity, similar to the seniority model of nuclear physics, which is a model for nuclear superconductivity.

\subsection{Glauber coherent states with color neutrality}

The condition for color neutrality reads

$$
\left\langle\Phi\left|S_{2}^{1}\right| \Phi\right\rangle=\left\langle\Phi\left|S_{3}^{2}\right| \Phi\right\rangle=\left\langle\Phi\left|S_{1}^{3}\right| \Phi\right\rangle=0, \quad\left\langle\Phi\left|S_{1}^{1}\right| \Phi\right\rangle=\left\langle\Phi\left|S_{2}^{2}\right| \Phi\right\rangle=\left\langle\Phi\left|S_{3}^{3}\right| \Phi\right\rangle,
$$

which implies

$$
\left|\alpha_{1}\right|=\left|\beta_{1}\right|,\left|\alpha_{2}\right|=\left|\beta_{2}\right|,\left|\alpha_{3}\right|=\left|\beta_{3}\right| .
$$

Then, we are led to minimize

$$
\mathcal{E}=G\left(\left|\alpha_{3}\right|^{2}\left(|\beta|^{2}+|\alpha|^{2}+2|\beta||\alpha|\right)+\left|\alpha_{3}\right|^{2}+3|\alpha|^{2}\right)
$$

with respect to $\left|\alpha_{3}\right|,|\alpha|,|\beta|$, under the constraints

$$
2 \Omega=2\left|\alpha_{3}\right|^{2}+|\alpha|^{2}+|\beta|^{2}, \quad 2 N-6 \Omega=3\left(|\alpha|^{2}-|\beta|^{2}\right) .
$$

This amounts to minimizing, with respect to $|a|$, the quantity

$\mathcal{E}=G\left[2|\alpha|^{2}+\frac{1}{3} N+2\left(\frac{1}{3} N-|\alpha|^{2}\right)\left(\Omega+|\alpha|^{2}-\frac{1}{3} N+\sqrt{|\alpha|^{2}\left(2 \Omega+|\alpha|^{2}-\frac{2}{3} N\right)}\right)\right]$.

The corresponding minimum is plotted, as a function of $N$, in Fig. 3. 


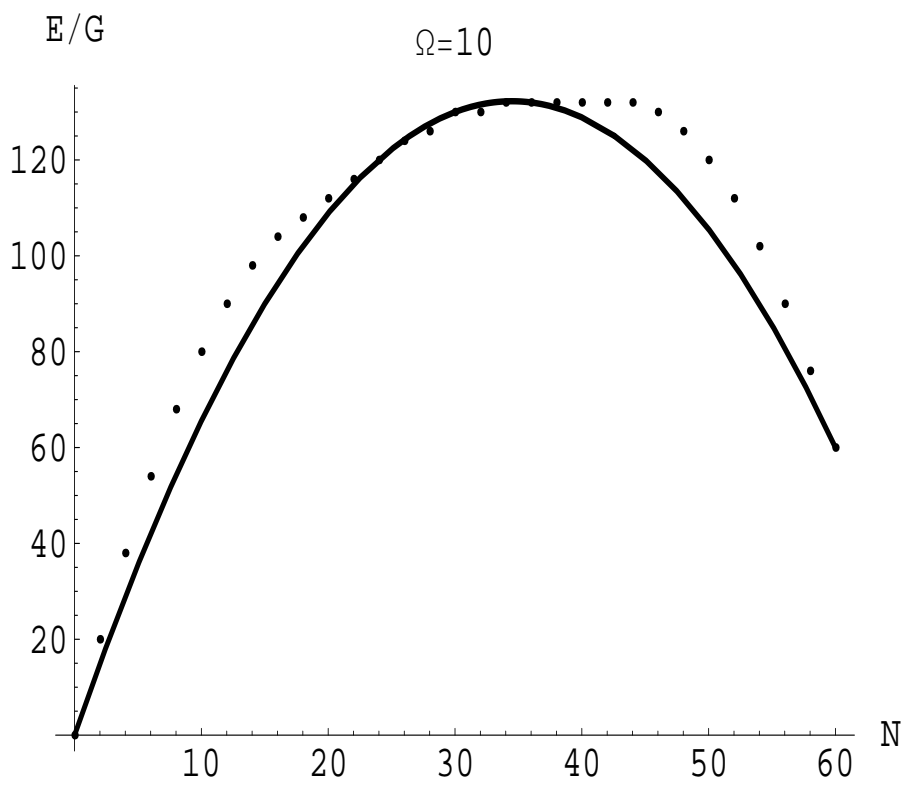

Figure 2: Exact results are shown. Absolute groundstate energy for even particle numbers (dots), and groundstate energy of the color neutral sector (continuous line) vs $N$, for $\Omega=10$. The continuous line is meaningful for values of $N$ multiples of 6 . For $N=30$ the groundstate is color symmetrical.

\section{Color-symmetrical states - exact treatment}

Consider the color symmetrical subspace spanned by the non-normalized kets

$$
|\Psi(m, \Lambda, \Gamma)\rangle=\left(\sum_{i} a_{i}^{\dagger} b_{i}^{\dagger}\right)^{m}\left(a^{\dagger} b^{\dagger}\right)^{\Lambda-m}\left(b^{\dagger}\right)^{\Gamma}|0\rangle,
$$

which are clearly invariant under $S U(3)$. This is an invariant subspace of $H$ of dimension $\Lambda+1$. From (7), (8) we have $2 \Omega=2 \Lambda+\Gamma, 2 N-6 \Omega=-3 \Gamma$. Moreover,

$$
\begin{aligned}
H|\Psi(m, \Lambda, \Gamma)\rangle= & G m(m+2)|\Psi(m-1, \Lambda, \Gamma)\rangle \\
& +G((2 \Lambda+\Gamma-2 m) m+(3 \Lambda-2 m))|\Psi(m, \Lambda, \Gamma)\rangle \\
& +G(\Lambda-m)(\Lambda+\Gamma-m)|\Psi(m+1, \Lambda, \Gamma)\rangle,
\end{aligned}
$$

where $m=0,1, \cdots, \Lambda$. This provides a $(\Lambda+1) \times(\Lambda+1)$ matrix representation of $H$ in the color-symmetrical sector. Numerically, the eigenvalues of the matrix so obtained may easily be determined. However, to obtain the groundstate energy, no diagonalization is required. We remark that the matrix which represents $G^{-1} H$ has non-negative entries and that the sum of the entries in any row is the same as for any other row. It is a well known result in matrix theory that this sum is the largest eigenvalue. In the present case the sum reads $m(m+2)+(\Lambda-m)(\Lambda+\Gamma-m)+$ $(2 \Lambda+\Gamma-2 m) m+(3 \Lambda-2 m)=\Lambda(\Lambda+\Gamma+3)$, independently of the value of $m$. It follows, therefore, that

$$
\mathcal{E}_{0}=G \Lambda(\Lambda+\Gamma+3)=G \frac{N}{3}\left(2 \Omega+3-\frac{1}{3} N\right)
$$

is the groundstate eigenvalue of the matrix representation of $H$, in the colorsymmetric subspace. It is the groundstate energy of the color-symmetrical sector 
for $3 \Omega \leq N \leq 6 \Omega$. We remark that the previous expression also holds for the sector $0 \leq N \leq 3 \Omega$, which may be similarly investigated by interchanging, in (9), $a^{\dagger}$ with $b^{\dagger}$.

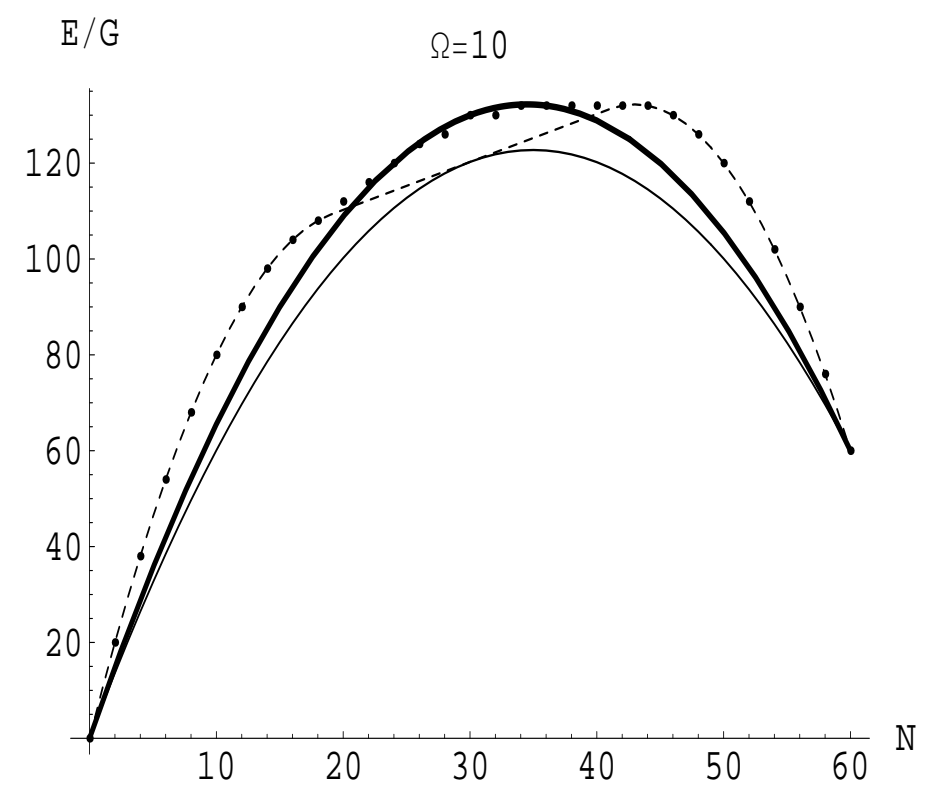

Figure 3: Exact groundstate energy for even particle numbers: dots. Glauber groundstate energy: dashed line. Lowest energy in the color neutral sector: thick line, which is meaningful for values of $N$ multiples of 6 . Lowest energy in the Glauber color neutral state: thin line. For $N \leq 2 \Omega$ and $N \geq 4 \Omega$, the exact result coincides with the Glauber result for the groundstate.

\section{$6 \quad$ Invariant sub-spaces of $H$}

The subspaces spanned by non-normalized kets of the any one of the following forms

$$
\begin{aligned}
\left|\Psi_{a a}(m, \Lambda, \Gamma, \Upsilon)\right\rangle & =\left(\sum_{i} a_{i}^{\dagger} b_{i}^{\dagger}\right)^{m}\left(a^{\dagger} b^{\dagger}\right)^{\Lambda-m}\left(a^{\dagger}\right)^{\Gamma}\left(a_{1}^{\dagger}\right)^{\Upsilon}|0\rangle, \\
\left|\Psi_{a b}(m, \Lambda, \Gamma, \Upsilon)\right\rangle & =\left(\sum_{i} a_{i}^{\dagger} b_{i}^{\dagger}\right)^{m}\left(a^{\dagger} b^{\dagger}\right)^{\Lambda-m}\left(a^{\dagger}\right)^{\Gamma}\left(b_{1}^{\dagger}\right)^{\Upsilon}|0\rangle, \\
\left|\Psi_{b a}(m, \Lambda, \Gamma, \Upsilon)\right\rangle & =\left(\sum_{i} a_{i}^{\dagger} b_{i}^{*}\right)^{m}\left(a^{\dagger} b^{\dagger}\right)^{\Lambda-m}\left(b^{\dagger}\right)^{\Gamma}\left(a_{1}^{\dagger}\right)^{\Upsilon}|0\rangle, \\
\left|\Psi_{b b}(m, \Lambda, \Gamma, \Upsilon)\right\rangle & =\left(\sum_{i} a_{i}^{\dagger} b_{i}^{\dagger}\right)^{m}\left(a^{\dagger} b^{\dagger}\right)^{\Lambda-m}\left(b^{\dagger}\right)^{\Gamma}\left(b_{1}^{\dagger}\right)^{\Upsilon}|0\rangle,
\end{aligned}
$$

where $m=0,1, \cdots, \Lambda$, are also invariant subspaces of $H$. Similar techniques to those used to obtain the groundstate energy of the color-symmetrical sector may be 


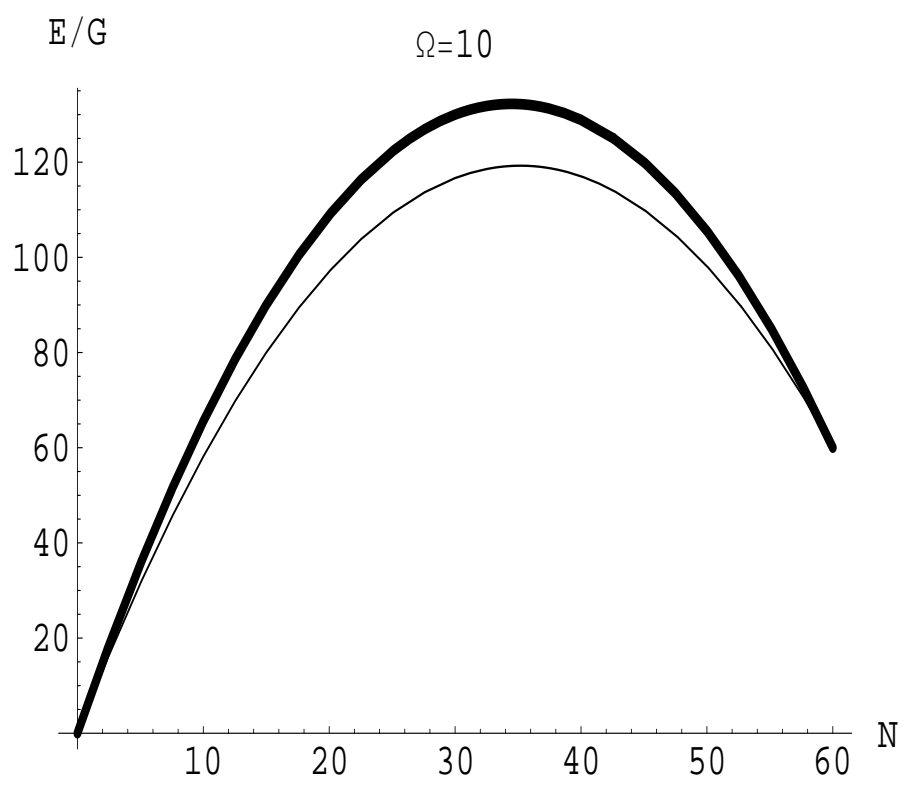

Figure 4: Groundstate energy of the color symmetrical sector versus the quark number, for $\Omega=10$. Thick line: exact result; thin line: color symmetrical BCS estimate .

used to obtain the groundstate energy in each invariant subspace. For instance, we have

$$
\begin{aligned}
& G^{-1} H\left|\Psi_{a a}(m, \Lambda, \Gamma, \Upsilon)\right\rangle=m(m+2+\Upsilon)\left|\Psi_{a a}(m-1, \Lambda, \Gamma, \Upsilon)\right\rangle \\
& +[(\Lambda-m)(2 m+\Upsilon)+m(\Gamma-2)+3 \Lambda+3 \Gamma+\Upsilon]\left|\Psi_{a a}(m, \Lambda, \Gamma, \Upsilon)\right\rangle \\
& +(\Lambda-m+\Gamma)(\Lambda-m)\left|\Psi_{a a}(m+1, \Lambda, \Gamma, \Upsilon)\right\rangle
\end{aligned}
$$

The sum of the above expansion coefficients is independent of $m$ and reads

$$
(\Lambda+1) \Upsilon+(\Lambda+\Gamma)(\Lambda+3) .
$$

In the invariant subspace spanned by the kets $\left|\Psi_{a a}(m, \Lambda, \Gamma, \Upsilon)\right\rangle$, the lowest energy is therefore $G[(\Lambda+1) \Upsilon+(\Lambda+\Gamma)(\Lambda+3)]$, the parameters $\Lambda, \Gamma, \Upsilon$, being restricted by the relations

$$
2 \Omega=2 \Lambda+\Gamma+\Upsilon, \quad 2 N-6 \Omega=3 \Gamma+\Upsilon .
$$

Combining the results obtained for each invariant subspace, the absolute groundstate energy is finally found. The results of this procedure are shown in Fig. 2, for $\Omega=10$. In Fig. 3, the results of the Glauber coherent state approach for $\Omega=10$, are also presented, and compared with the exact results. The groundstate is not color-symmetrical, except for $N=3 \Omega$. Thus, in general, the groundstate is not made up of color neutral triplets. We remark that the lowest energy curve of the color neutral sector is "tangent" to the absolute groundstate energy curve. Similarly, for the Glauber coherent state, the lowest energy curve of the color neutral sector is also tangent to the absolute groundstate energy curve, in the corresponding approximation.

In Fig. 4 we illustrate the performance of the color-symmetric BCS approach developed in [4] for the description of the color-symmetric sector of the model, being

$$
\mathcal{E}_{B C S}=\frac{G N}{9}\left(6 \Omega-N+1+\frac{4 N}{3 \Omega}\right),
$$

the color-symmetric groundstate energy in that approximation [4]. 


\section{Conclusions}

Exact solutions are presented to the equations of the lowest energy states of the colored and color-symmetrical sectors of the $S U(3)$ symmetric Bonn quark model, which is defined in terms of an effective pairing force with $s u(4)$ algebraic structure. It is shown that the groundstate of the model belongs to the unphysical color symmetry breaking sector, except for a narrow interval in the range of admissible quark numbers. The exact treatment of the model was carried out in the framework of the Schwinger realization of the $s u(4)$ algebra. The performances of the Glauber coherent state and of the BCS theory with respect to the description, not only of the absolute (colored) groundstate, but also of the minimum energy state of the color-symmetrical sector, have been studied, it being found that they are remarkably good. However, the description of the color-symmetrical sector requires proper color-symmetric versions of the Glauber coherent state and of the BCS theory.

\section{Acknowledgments}

The present research was partially supported by projects POCI/FP/81923/2007 and CERN/FP/ 83505/2008.

\section{References}

[1] H.R. Petry, H. Hofstaedt, S. Merk, K. Bleuler, H. Bohr and K.S. Narain, Phys. Lett. B159 (1985)363.

[2] P. Ring and P. Schuk, The nuclear many-body problem, Springer-Vrlag, NewYork, Heidelberg, Berlin, 1980.

[3] S. Pittel, J. Engel, J. Dukelsky and P. Ring, Phys. Lett. B247 (1990) 185.

[4] H. Bohr and J. da Providência, J. Phys. A: Math. Theor. 41 (2008) 405202; H. Bohr and J. da Providência, J. Phys. A: Math. Theor. 42 (2009) 089802, corrigendum.

[5] M. Alford, K. Rajagopal, S. Reddy and F. Wilczek, Phys. Rev. D64 (2001) 074017; K. Rajagopal and F. Wilczek, Phys. Rev. Lett. 86 (2001) 3492; M.G. Alford, Annu. Rev. Nucl. Part. Sci. 51 (2001) 131; M. Alford and S. Reddy, Phys. Rev. D67 (2003) 074024.

[6] M.G. Alford, K. Rajagopal, T. Shaefer, A. Schmitt, Rev. Mod. Phys. 80 (2008) $1455-1515$.

[7] K. Iida and G. Baym, Phys. Rev. D63 (2001) 074018; M. Alford and M. Rajagopal, J. High Energy Phys. 06 (2002) 031; A.W. Steiner, S. Reddy and M. Prakash, Phys. Rev. D66 (2002)094007; M. Huang, P. Zhuang and W. Chao, Phys. Rev. D67 (2003) 065015.

[8] M. Yamamura, T. Kuriyama, A. Kunihiro, Prog. Theor. Phys. 104 (2000) 385. 